\title{
Antiradical and antimicrobial properties of fermented red chicory (Cichorium intybus L.) by-products
}

\author{
Dafni Maria Kagkli ${ }^{1}$ - Viviana Corich ${ }^{1} \cdot$ Barbara Bovo $^{1} \cdot$ Anna Lante $^{1}$. \\ Alessio Giacomini ${ }^{1}$
}

Received: 10 March 2015 / Accepted: 30 May 2016/Published online: 10 June 2016

(C) Springer-Verlag Berlin Heidelberg and the University of Milan 2016

\begin{abstract}
Discarded leaves of red chicory (Radicchio "Rosso di Chioggia" IGP) were fermented with one Saccharomyces yeast and four lactic acid bacteria chosen on the basis of their ability to grow on plant material without any need of supplements. Antiradical and antimicrobial activities of the resulting products were assessed. Among the strains tested, Lactobacillus plantarum and L. hilgardii gave the best performances and also provided fermented substrates with antiradical and antimicrobial activities. In particular the latter compounds were found only in fermented samples, confirming that the choice of appropriate microorganisms for fermentation could be useful when the aim is to target specific functional foods starting from by-products or waste material.
\end{abstract}

Keywords Cichorium intybus · By-products · Fermentation · Antioxidant/antiradical activity · Antimicrobial activity ·

Functional food

\section{Introduction}

The genus Cichorium includes four wild species (C. bottae, C. spinosum, C. calvum, and C. pumilum) and two widely

Dafni Maria Kagkli and Viviana Corich are joint first authors.

Dafni Maria Kagkli and Viviana Corich contributed equally to this work.

Alessio Giacomini

alessio.giacomini@unipd.it

1 Department of Agronomy, Food, Natural Resources, Animals and the Environment (DAFNAE), University of Padova, viale dell'Università 16, Legnaro, Padova 35020, Italy cultivated ones, namely $C$. intybus (chicory) and $C$. endivia (lettuce). Chicory is a Mediterranean vegetable that is also widespread throughout Europe, Western Asia and North America, including several varieties with leaves varying in color from white to dark red. Some cultivars are seasonal, while other are produced continuously throughout the year, such as the red chicory "Radicchio rosso di Chioggia. Commercial production of colored radicchio varieties involves a laborious procedure that includes the removal of many outer leaves from heads, with the aim of keeping the inside that are better developed and characteristic of the product. This procedure generates large amounts of by-products, the disposal of which represents a relevant environmental problem, not the least due to the presence of phenolic compounds (Arvanitoyannis and Kassaveti 2007).

Historically, chicory was used by the ancient Egyptians as a medicinal plant, and different parts of this plant are still utilized in folk medicines to treat various symptoms (Street et al. 2013). In the food industry chicory is considered to be a prebiotic because it is rich in minerals and inulin. Studies conducted to date have revealed the antioxidant potentialities of this vegetable (Innocenti et al. 2005; Rossetto et al. 2005; Koukounaras and Siomos 2010; Ferioli et al. 2015; Finotti et al. 2015) which are linked to its high content of phenolic compounds. Nevertheless, as yet chicory has not been shown to possess antimicrobial activity and, to our knowledge no study has investigated the ability of lactic acid bacteria to grow in this vegetable medium, possibly increasing its beneficial properties.

Fermentation by lactic acid bacteria (LAB) can be considered a simple and valuable method to preserve or even improve the safety, sensory and nutritional aspects of food products. Numerous LAB strains are routinely used as starter cultures in the dairy, meat and bakery industries, but a much more limited number have been used for fermentation of vegetables, 
among which Lactobacillus plantarum is the LAB starter most frequently used commercially (Ruiz-Barba et al. 1994; Leroy and De Vuyst 2004). This species is commonly part of the autochthonous microbiota of raw vegetables and together with Lactobacillus hilgardii, Oenococcus oeni, Lactobacillus brevis and Leuconostoc mesenteroides is frequently isolated from grape musts and other plant material (Moreno-Arribas et al. 2003; Rodas et al. 2005).

In the study reported here we tested several microorganisms for fermenting "Radicchio Rosso di Chioggia" leaves to assess the production of potential by-products with antiradical and antimicrobial activities.

\section{Materials and methods}

\section{Strains and growth conditions}

Strains used for fermentations and bacteria used as indicators of antimicrobial activity are listed in Table 1. Autochthonous strains of L. hilgardii and L. plantarum were isolated from plant material (Maragkoudakis et al. 2013).

Lactobacillus delbrueckii subsp. bulgaricus, L. hilgardii and L. plantarum were grown in de Man, Rogosa and Sharp medium (MRS; Oxoid Ltd., Basingstoke, UK), Streptococcus thermophilus in M17 medium (Oxoid), Staphylococcus xylosus in Corynebacterium Broth (Oxoid), Listeria innocua and Escherichia coli in Brain Heart Infusion (BHI; Oxoid) and Bacillus amyloliquefaciens, B. subtilis and Pseudomonas fluorescens in Nutrient Broth (NB; Oxoid). L. delbrueckii subsp. bulgaricus, St. thermophilus and E. coli were incubated at $37^{\circ} \mathrm{C}$; the other strains were incubated at $30^{\circ} \mathrm{C}$. Saccharomyces cerevisiae was grown in Yeast Mould Broth (YMB; Oxoid) at $30^{\circ} \mathrm{C}$ for $24 \mathrm{~h}$.

For preparation of inocula $8 \mathrm{ml}$ of each culture at the stationary phase (approx. $10^{8} \mathrm{CFU} / \mathrm{ml}$ ) was centrifuged for $5 \mathrm{~min}$ at $5000 \mathrm{~g}$ to remove the supernatant and then resuspended in the same volume of saline solution $(0.9 \% \mathrm{NaCl}$ w/v). For each cell suspension proper dilutions (approx. 1:1000) were performed in order to standardize the inoculum. Plate counts were performed to confirm the inoculum size.

Determination of autochthonous microbiota of chicory was performed by plate count on Plate Count Agar (PCA) medium (Oxoid) after $24 \mathrm{~h}$ of incubation at $30^{\circ} \mathrm{C}$.

\section{Fermentation of red chicory leaves}

Fresh heads of Radicchio "Rosso di Chioggia" were purchased from the local market and washed thoroughly with tap water. A 200-g sample of leaves was then mixed with $0.9 \% \mathrm{NaCl}$ at a ratio of $1: 1(\mathrm{w} / \mathrm{v})$ and homogenized for $2 \mathrm{~min}$ with a blender. Aliquots of $30 \mathrm{~g}$ were rapidly transferred to $50-\mathrm{ml}$ sterile Falcon tubes, inoculated with the appropriate microorganism to a final cell density of about $10^{5} \mathrm{CFU} / \mathrm{ml}$ of homogenate and incubated at $30{ }^{\circ} \mathrm{C}$ for $48 \mathrm{~h}$. A tube without inoculum was also incubated as control. All tests were performed in triplicate.

The samples were pasturized by immersing the Falcon tubes containing the inoculum and microorganism in a water bath at $72{ }^{\circ} \mathrm{C}$ for $15 \mathrm{~min}$, followed by cooling at room temperature.

The $\mathrm{pH}$ values were measured after $0,4,24$ and $48 \mathrm{~h}$ by immersing the $\mathrm{pH}$-meter electrode directly into the tubes.

\section{Freeze-drying of culture supernatants}

When the $\mathrm{pH}$ of the fermenting cultures reached a steady value, the tubes were centrifuged at $5000 \mathrm{~g}$ for $15 \mathrm{~min}$, and each supernatant was transferred into a new sterile Falcon tube and immediately freeze-dried. The powder was stored at $-80{ }^{\circ} \mathrm{C}$ for later use. For the experiments, the powder was dissolved in sterile water at a final concentration of $200 \mathrm{mg} / \mathrm{ml}$.

\section{Antimicrobial activity assay}

The well diffusion technique (Giacomini et al. 2000; Drosinos et al. 2007) was used to evaluate antimicrobial activity against the indicator bacterial strains reported in Table 1 . The freezedried powders were used at final concentration of $4 \mathrm{mg} / \mathrm{ml}$. With this technique, the formation of inhibition haloes surrounding the wells indicates the presence of activity, where the diameter of the haloes is related to the amount of inhibitory substance.

The proteinaceous nature of the inhibitory substance was checked by treating the extract with proteinase $\mathrm{K}(1 \mathrm{mg} / \mathrm{ml}$; Sigma-Aldrich Corp., St. Louis, MO) before use and then looking for absence of inhibitory activity on the culture plates.

\section{Determination of total polyphenol content and antioxidant activity}

Total polyphenol content was measured using the FolinCiocalteau method (Singleton et al. 1999), with the concentrations expressed as gallic acid equivalents (GAE; Escarpa and Gonzales 2001) based on comparison against the gallic acid calibration curve $\left(R^{2} 0.9049\right)$.

The DPPH (2,2-diphenyl-1-picrylhydrazyl; Sigma-Aldrich Ltd.) assay was used to determine the antiradical activity of the extracts, according to Miliauskas et al. (2004). Radical scavenging activity was calculated by the formula:

$\%$ of inhibition $=\left(A_{B}-A_{A}\right) / A_{B} \times 100$ 
Table 1 Bacterial strains used in the study

\begin{tabular}{|c|c|}
\hline Strain & Source \\
\hline \multicolumn{2}{|l|}{ Strains used for fermentations } \\
\hline Lactobacillus delbrueckii subsp. bulgaricus DSM $20081^{\mathrm{T}}$ & $\operatorname{DSMZ}(*)$ \\
\hline Lactobacillus hilgardii DSM $20176^{\mathrm{T}}$ & DSMZ \\
\hline Lactobacillus hilgardii TGMMRS06 & Maragkoudakis et al. 2013 \\
\hline Lactobacillus hilgardii TGMMRS08 & Maragkoudakis et al. 2013 \\
\hline Lactobacillus hilgardii TGMMRS11 & Maragkoudakis et al. 2013 \\
\hline Lactobacillus hilgardii TGMMRS12 & Maragkoudakis et al. 2013 \\
\hline Lactobacillus hilgardii TGMMRS13 & Maragkoudakis et al. 2013 \\
\hline Lactobacillus hilgardii TGMMRS25 & Maragkoudakis et al. 2013 \\
\hline Lactobacillus hilgardii TGMMRS27 & Maragkoudakis et al. 2013 \\
\hline Lactobacillus hilgardii TGMMRS35 & Maragkoudakis et al. 2013 \\
\hline Lactobacillus hilgardii TGMMRS41 & Maragkoudakis et al. 2013 \\
\hline Lactobacillus plantarum DSM $20174^{\mathrm{T}}$ & DSMZ \\
\hline Lactobacillus plantarum Т30РСР06 & Maragkoudakis et al. 2013 \\
\hline Lactobacillus plantarum Т30РCР07 & Maragkoudakis et al. 2013 \\
\hline Lactobacillus plantarum T30РCP14 & Maragkoudakis et al. 2013 \\
\hline Lactobacillus plantarum Т30РCР05 & Maragkoudakis et al. 2013 \\
\hline Lactobacillus plantarum Т30РCР01 & Maragkoudakis et al. 2013 \\
\hline Lactobacillus plantarum T4MPCP71 & Maragkoudakis et al. 2013 \\
\hline Lactobacillus plantarum T4MPCP69 & Maragkoudakis et al. 2013 \\
\hline Lactobacillus plantarum T4MPCP77 & Maragkoudakis et al. 2013 \\
\hline Lactobacillus plantarum T4MPCP78 & Maragkoudakis et al. 2013 \\
\hline Lactobacillus plantarum T4MPCP73 & Maragkoudakis et al. 2013 \\
\hline Saccharomyces cerevisiae DSM $70449^{\mathrm{T}}$ & DSMZ \\
\hline Streptococcus thermophilus DSM $20617^{\mathrm{T}}$ & DSMZ \\
\hline \multicolumn{2}{|l|}{ Strains used as indicators } \\
\hline Bacillus amyloliquefaciens DSM $7^{\mathrm{T}}$ & DSMZ \\
\hline Bacillus subtilis DSM $10^{\mathrm{T}}$ & DSMZ \\
\hline Escherichia coli DSM $30083^{\mathrm{T}}$ & DSMZ \\
\hline Listeria innocua DSM $20649^{\mathrm{T}}$ & DSMZ \\
\hline Pseudomonas fluorescens DSM $50090^{\mathrm{T}}$ & DSMZ \\
\hline Staphylococcus xylosus DSM $20266^{\mathrm{T}}$ & DSMZ \\
\hline
\end{tabular}

DSMZ, Deutsche Sammlung von Mikroorganismen und Zellkulturen GmbH where $A_{\mathrm{B}}$ represents the absorption of blank sample ( $t=0 \mathrm{~min}$ ) and $A_{\mathrm{A}}$ is the absorption of the tested extract solution $(t=15 \mathrm{~min})$.

Ascorbic acid at different concentrations was used as the control. All experiments were performed in triplicate.

\section{Determination of phenolic acids and glucose}

Phenolic acids were determined according to Rossetto et al. (2005) using a high-performance liquid chromatography (HPLC;Thermo-Finnigan, San Jose, CA ) equipped with a photodiode array detector (UV 6000LP). All samples were filtered on a $0.45-\mu \mathrm{m}$ cartridge and directly injected. The phenolic acids were separated using an
LC-18 Suppelco-sil column (Sigma-Aldrich Ltd.) at $25{ }^{\circ} \mathrm{C}$ with a flow rate of $1.5 \mathrm{ml} / \mathrm{min}$ under isocratic conditions. The mobile phase consisted of an aqueous solution containing $1.5 \%$ acetic acid and $18 \% n$-butanol. Phenolic acid standards were passed through the column both singly and in mixture. The spectrum of each standard was recorded and stored in the HPLC library data. Each reported value was the average of three repeated analyses. The internal and external standards included to identify the molecules on the basis of their UV spectrum and retention time were those reported by Lante et al. (2011). In brief, we compared spectral data of sample peaks with those obtained for the phenolic acids standard to confirm the reliability of the identification since matches between 
the spectra of the samples and those of their standards showed a $\geq 90 \%$ match.

D-Glucose content of chicory homogenates was determined by HPLC as reported by Zuleta and Sambucetti (2001). The HPLC system and conditions were as follows: an Aminex HPX-87C (Bio-Rad, Hercules, CA) anionexchange column, deionized water at $85{ }^{\circ} \mathrm{C}$ as the mobile phase and a flux rate of $0.6 \mathrm{~mL} / \mathrm{min}$. To identify the sugars present in the samples, we used reference solutions of monoand disaccharides.

\section{Results and discussion}

\section{Fermentation of chicory homogenates}

Red chicory homogenates were used as the growth substrate for type strains of one yeast $\left(\right.$ S. cerevisiae DSM $\left.70449^{\mathrm{T}}\right)$ and four LAB (L. delbrueckii subsp. bulgaricus DSM $20081^{\mathrm{T}}$, L. hilgardii DSM $20176^{\mathrm{T}}$, L. plantarum DSM $20174^{\mathrm{T}}$ and St. thermophilus DSM $20617^{\mathrm{T}}$ ), chosen among species frequently associated with the production of fermented foods. Cultures of these strains were used to inoculate chicory homogenates at about $10^{5} \mathrm{CFU} / \mathrm{g}$, which was considered a sufficiently large inoculum to outcompete the autochthonous microbiota of chicory (Bovo et al. 2011), which was determined to be $4.0 \times 10^{4} \mathrm{CFU} / \mathrm{g}$.

\section{Microbial growth}

Inoculated chicory homogenates were incubated for $48 \mathrm{~h}$ to allow a full development of the bacterial population and therefore maximize the production and possible release of compounds of interest. Microbial growth in the chicory cultures was measured by plate counting after $48 \mathrm{~h}$ of incubation (Table 2). All strains tested were able to grow on the chicory homogenates, albeit at different levels. All four Lactobacillus species grew to $>10^{9} \mathrm{CFU} / \mathrm{ml}$ while St. thermophilus and $S$. cerevisiae growth just slightly exceeded $10^{7} \mathrm{CFU} / \mathrm{ml}$, a value lower than that of the non-inoculated sample, which reached $1.2 \times 10^{8} \mathrm{CFU} / \mathrm{g}$. One explanation for this approximately 1-log decrease is that it is the result of an unknown negative interaction or competition between the inoculated strains and the autochthonous microbiota that negatively affected the overall microbial growth.

\section{$p H$}

The starting $\mathrm{pH}$ of the chicory homogenate was 5.7. All lactobacilli were able to lower the $\mathrm{pH}$ by more than $2 \mathrm{pH}$ units after $48 \mathrm{~h}$ of incubation, to values ranging from 3.3 to 3.5 (Table 2). This low value creates a selective environment that could explain the high population numbers reached by all of the strains of this species with respect to the autochthonous microbiota. The $\mathrm{pH}$ of the matrix inoculated with $S t$. thermophilus did not go below $\mathrm{pH} 4.5$, which is in accordance with its known lower acidifying capability with respect to that of lactobacilli, while $S$. cerevisiae, which does not perform lactic acid fermentation, did not lower the $\mathrm{pH}$ significantly, although it is known its potentiality to produce variable amounts of organic acids under specific conditions (Martìnez-Muñoz and Kane 2008). The autochthonous microbiota in the non-inoculated substrate brought the $\mathrm{pH}$ down to 4.6 , indicating the presence of acidifying microbes, probably $\mathrm{LAB}$, which are normally present in good amounts on plant material.

\section{Scavenging capacity and antimicrobial activity}

The radical scavenging activity of the strains was assessed (Tables 2, 3), with L. hilgardii and L. plantarum giving the best results, well above the other three strains which showed activities just slightly higher than that of the non-inoculated control. As recently reported by Hur et al. (2014), the total antioxidative activity of a fermented plant extract is based on the synergism between polyphenolic compounds and/or other factors, such as microorganisms. A possible explanation is that these LAB have their own antioxidative activities to protect themselves against oxidative damage. As a result, fermenting bacteria might increase the synthesis of the major non-enzymatic antioxidants and free radical scavengers such as glutathione or catalase, as suggested by Spyropoulos et al. (2011).

Antimicrobial activity of fermented plant extract was tested against six indicator strains, namely $B$. amiloliquefaciens, B. subtilis, E. coli, Listeria innocua, P. fluorescens and Staph. xylosus, chosen among species/strains closely related to pathogenic or spoilage bacteria. Well diffusion tests with supernatants of fermented cultures did not produce visible haloes on the disk plates against any of the indicators tested, revealing the absence of antimicrobial activity towards the chosen indicators, at least at the concentrations applied.

\section{Fermentation of pasteurized chicory homogenates}

In order to reduce possible interferences on growth and activity of the introduced strains, all fermentation tests were also performed on pasteurized chicory homogenates (Table 2).

\section{Microbial growth}

As expected, the heat treatment heavily affected the resident microbiota (Table 2). Consequently, after $48 \mathrm{~h}$ in the noninoculated sample, the population, probably composed of thermoduric species, reached levels about 50-fold lower than that of the non-pasteurized samples. This decrease indeed did 
Table 2 Growth, $\mathrm{pH}$, scavenging activity of the DPPH radical and glucose content of cultures developed in chicory homogenates after $48 \mathrm{~h}$ of culture

\begin{tabular}{|c|c|c|c|c|}
\hline Strain & $\mathrm{CFU} / \mathrm{g}$ & $\mathrm{pH}$ & $\begin{array}{l}\text { Scavenging } \\
\text { activity (\%) }\end{array}$ & $\begin{array}{l}\text { Glucose } \\
\text { concentration }(\mathrm{g} / \mathrm{l})\end{array}$ \\
\hline \multicolumn{5}{|l|}{ Non-pasteurized chicory } \\
\hline Raw red chicory homogenate ${ }^{a}$ & $4.0 \times 10^{4} \pm 8.0 \times 10^{3}$ & 5.7 & $10.3 \mathrm{a}$ & ND \\
\hline Non-inoculated control & $1.2 \times 10^{8} \pm 1.1 \times 10^{7}$ & 4.6 & $14.5 \mathrm{a}, \mathrm{b}$ & $1.33 \pm 0.037$ \\
\hline S. cerevisiae DSM $70449^{\mathrm{T}}$ & $1.0 \times 10^{7} \pm 3.3 \times 10^{6}$ & 5.1 & $17.6 \mathrm{~b}$ & \\
\hline St. thermophilus DSM $20617^{\mathrm{T}}$ & $2.2 \times 10^{7} \pm 4.0 \times 10^{6}$ & 4.5 & $18.1 \mathrm{~b}$ & \\
\hline L. delbrueckii DSM $20081^{\mathrm{T}}$ & $3.0 \times 10^{9} \pm 6.4 \times 10^{8}$ & 3.5 & $18.1 \mathrm{~b}$ & \\
\hline L. hilgardii DSM $20176^{\mathrm{T}}$ & $4.0 \times 10^{9} \pm 5.5 \times 10^{8}$ & 3.3 & $37.8 \mathrm{c}$ & $2.73 \pm 0.037$ \\
\hline L. plantarum DSM $20174^{\mathrm{T}}$ & $4.0 \times 10^{9} \pm 7.1 \times 10^{8}$ & 3.3 & $36.8 \mathrm{c}$ & $2.55 \pm 0.005$ \\
\hline \multicolumn{5}{|l|}{ Pasteurized chicory } \\
\hline Raw red chicory homogenate ${ }^{a}$ & $1.4 \times 10^{3} \pm 8.0 \times 10^{2}$ & 5.2 & $17.6 \mathrm{a}$ & ND \\
\hline Non-inoculated control & $1.0 \times 10^{6} \pm 5.5 \times 10^{5}$ & 5.0 & $19.6 \mathrm{a}$ & ND \\
\hline S. cerevisiae DSM $70449^{\mathrm{T}}$ & $8.5 \times 10^{7} \pm 1.6 \times 10^{6}$ & 4.2 & $12.4 \mathrm{a}, \mathrm{b}$ & \\
\hline St. thermophilus DSM $20081^{\mathrm{T}}$ & $2.0 \times 10^{7} \pm 4.9 \times 10^{6}$ & 4.5 & $15.7 \mathrm{a}$ & \\
\hline L. delbrueckii DSM $20081^{\mathrm{T}}$ & $2.9 \times 10^{9} \pm 8.5 \times 10^{8}$ & 3.6 & $17.3 \mathrm{a}$ & \\
\hline L. hilgardii DSM $20176^{\mathrm{T}}$ & $4.1 \times 10^{9} \pm 2.6 \times 10^{8}$ & 3.2 & $37.6 \mathrm{c}$ & $2.60 \pm 0.04$ \\
\hline L. plantarum DSM $20174^{\mathrm{T}}$ & $4.3 \times 10^{9} \pm 1.9 \times 10^{8}$ & 3.3 & $32.8 \mathrm{c}$ & $3.22 \pm 0.022$ \\
\hline
\end{tabular}

Data are reported as the mean of triplicate readings/measurements \pm standard deviation (SD) or as the mean without SD. Values followed by the same lowercase letter do not differ at $p<0.05$

DPPH, 2,2-Diphenyl-1-picrylhydrazyl; ND, none detected

${ }^{\mathrm{a}}$ Measured at the beginning of the test not appear to have an evident impact on the growth of the inoculated strains that reached levels comparable to those of the untreated samples, with the sole exception of $S$. cerevisiae that increased its level by about $1 \log$.

pH

The heat treatment influenced the initial $\mathrm{pH}$ of the homogenate, with an initial decrease to 5.2 , followed by a decrease to 5.0 and stabilization after $48 \mathrm{~h}$ (Table 2b). In comparison, the final $\mathrm{pH}$ of the samples inoculated with bacteria reflected the values of the corresponding non-pasteurized cultures. This is not surprising, since in both cases population levels were similarly high. The growth of S. cerevisiae determined a decrease in $\mathrm{pH}$ of nearly $1 \mathrm{pH}$ unit with respect to the untreated chicory homogenate. At conditions of very low $\mathrm{pH}$, microbial competition generally favors yeasts, which are more tolerant to acidic $\mathrm{pH}$, leading quickly to the domination of yeasts in the culture (Ribéreau-Gayon et al. 2006). In our study, the resident microbiota seems to have negatively affected the inoculated yeast much more than bacteria, probably due to the relatively high $\mathrm{pH}$.

\section{Antiradical and antimicrobial activities}

Radical scavenging activity of all fermented pasteurized substrates was determined (Table 2). Our results do not substantially differ from those of non-pasteurized samples as
L. hilgardii and L. plantarum still proved to be the best performing species, with about twice the level of radical scavenging activity as the other strains tested. Therefore, the previously proposed hypothesis of a synergism between polyphenolic compounds and microorganism species seems also to fit our data.

In terms of antimicrobial activity, the supernatants of fermented cultures did not produce haloes on the culture plates, thus demonstrating the absence of antimicrobial activity, at least at the concentrations used.

\section{Fermentation with autochthonous isolates of $L$. hilgardii and $L$. plantarum}

Since L. hilgardii and L. plantarum showed the strongest scavenging capabilities of the LAB tested, we investigated these species in more detail. L. hilgardii is largely found in wines, and many strains are known to exhibit a high alcohol tolerance and a capability to withstand low $\mathrm{pH}$ and harsh conditions (Rodas et al. 2005). Its capability to grow well in the presence of polyphenols has also been reported (Alberto et al. 2001). Lactobacillus plantarum strains are widespread on plant material. In food processing systems they are frequently used for technological transformations, including the malolactic fermentation of wines (Miller et al. 2011), and are exploited as starter cultures for the fermentation of vegetables and fruits, such as olives, cabbages, carrots and beets (Gardner 
et al. 2001; Rodríguez et al. 2009). They are also recovered from meat and dairy products (Vuyst and Vandamme 1994).

Ten L. hilgardii (9 autochthonous isolates and the type strain) and $11 \mathrm{~L}$. plantarum (10 autochthonous isolates and the type strain) strains were used in our study for the fermentation of pasteurized chicory homogenates (Table 3 ).

\section{Growth and $p H$ evolution}

The growth of the L. hilgardii strains in pasteurized chicory is reported in Table 3 . The $\mathrm{pH}$ was measured throughout a period of $48 \mathrm{~h}$. Six strains lowered the $\mathrm{pH}$ to values ranging from 3.7 to 3.9, and three strains reached values of between 4.1 and 4.2. The $\mathrm{pH}$ of the pasteurized chicory homogenate cultured with strain TGMMRS06 reached 3.9 just after $24 \mathrm{~h}$, indicating that this strain was the fastest acidifying strain among those tested. The type strain grew significantly better than all natural isolates since it lowered the $\mathrm{pH}$ to 3.5 just after $3 \mathrm{~h}$; this efficiency may be related to its origin, since it was isolated from wine, which has $\mathrm{pH}$ values of less than 3.5.
The $\mathrm{pH}$ of $L$. plantarum cultures was measured throughout a period of $48 \mathrm{~h}$ (Table 3). All isolates brought the $\mathrm{pH}$ to values well below those of $L$. hilgardii, ranging from 3.2 to 3.5. Moreover, all strains were able to lower the $\mathrm{pH}$ to $\leq 4.0$ just after $4 \mathrm{~h}$ of incubation. The behavior of the type strain was similar to that of the natural isolates.

These results clearly demonstrate that $L$. plantarum strains have on average a considerable stronger and faster acidifying capability than L. hilgardii. The $\mathrm{pH}$ of the non-inoculated pasteurized control chicory remained at $\mathrm{pH} 5.2$ throughout the incubation period.

\section{Antimicrobial activity}

No antimicrobial activity was observed for all natural isolates of $L$. hilgardii and $L$. plantarum using culture supernatants for the well diffusion assays. Consequently, we concentrated the samples by freeze-drying in order to check possible antimicrobial activity of molecules present at very low concentrations.
Table $3 \mathrm{pH}$ evolution and scavenging activity of the DPPH radical by $L$. hilgardii and L. plantarum in pasteurized chicory samples

\begin{tabular}{|c|c|c|c|c|c|}
\hline \multirow[t]{2}{*}{ Sample } & \multicolumn{4}{|c|}{$\mathrm{pH}$ value after: } & \multirow{2}{*}{$\begin{array}{l}\text { Scavenging activity } \\
(\%) \text { at } 48 \mathrm{~h}^{\mathrm{a}}\end{array}$} \\
\hline & $0 \mathrm{~h}$ & $4 \mathrm{~h}$ & $24 \mathrm{~h}$ & $48 \mathrm{~h}$ & \\
\hline Control & 5.5 & 5.2 & 5.2 & 5.2 & $8.15 \pm 0.39$ \\
\hline \multicolumn{6}{|l|}{ L. hilgardii } \\
\hline DSM 20176 & & 3.5 & 3.3 & 3.3 & $37.8 \pm 1.89$ \\
\hline TGMMRS06 & & 5.3 & 3.9 & 3.8 & $8.77 \pm 0.44$ \\
\hline TGMMRS08 & & 5.3 & 4.5 & 3.8 & $2.61 \pm 0.13$ \\
\hline TGMMRS11 & & 5.4 & 4.5 & 3.8 & ND \\
\hline TGMMRS12 & & 5.2 & 4.7 & 3.8 & ND \\
\hline TGMMRS13 & & 5.3 & 4.8 & 4.1 & $6.22 \pm 0.31$ \\
\hline TGMMRS25 & & 5.3 & 4.8 & 4.2 & $18.12 \pm 0.91$ \\
\hline TGMMRS27 & & 5.1 & 4.5 & 3.9 & $\mathrm{ND}$ \\
\hline TGMMRS35 & & 5.4 & 4.4 & 3.7 & $7.43 \pm 0.37$ \\
\hline TGMMRS41 & & 5.3 & 4.6 & 4.1 & ND \\
\hline \multicolumn{6}{|l|}{ L. plantarum } \\
\hline DSM 20174 & & 3.6 & 3.2 & 3.2 & $36.8 \pm 1.84$ \\
\hline Т30РСР01 & & 3.8 & 3.4 & 3.2 & $24.34 \pm 1.22$ \\
\hline Т30РСР05 & & 4.1 & 4.1 & 3.4 & $24.76 \pm 1.24$ \\
\hline Т30РСР06 & & 4.1 & 4.1 & 3.3 & $21.89 \pm 1.10$ \\
\hline Т30РСР07 & & 3.9 & 3.9 & 3.3 & $25.80 \pm 1.29$ \\
\hline Т30РCP14 & & 4.1 & 4.2 & 3.2 & $10.11 \pm 0.51$ \\
\hline Т4MРCP69 & & 3.8 & 3.8 & 3.5 & $8.12 \pm 0.41$ \\
\hline T4MPCP71 & & 4.0 & 4.0 & 3.4 & $25.95 \pm 1.30$ \\
\hline T4MPCP73 & & 3.9 & 4.0 & 3.4 & $24.45 \pm 1.22$ \\
\hline T4MPCP77 & & 4.2 & 4.1 & 3.4 & $24.46 \pm 1.23$ \\
\hline Т4MPCP78 & & 4.0 & 4.0 & 3.4 & $22.48 \pm 1.12$ \\
\hline
\end{tabular}

ND: none detected

${ }^{\text {a }}$ Scavenging activity is presented as the mean $\pm \mathrm{SD}$ of triplicate measurements 
Aliquots of freeze-dried powder $(10 \mathrm{mg})$ of all L. hilgardii and $L$. plantarum fermented substrates were tested against six indicator strains, namely B. amiloliquefaciens, B. subtilis, E. coli, Listeria innocua, P. fluorescens and St. xylosus (Table 4).

The control culture did not show any antimicrobial activity against any of the indicator strains, while the inoculated substrates gave differentiated responses.

Supernatant of the type strains of both species tested showed the strongest antimicrobial activity against all of the indicators used.

Some L. plantarum and L. hilgardii strains did not inhibit any of the indicators, while others were effective against one or more. In particular, L. hilgardii TGMMRS08 and TGMMRS12 inhibited five of the six indicators and L. plantarum $\mathrm{T} 30 \mathrm{PCP} 01$ inhibited all of the indicators tested.

The L. hilgardii strains shared some common behaviors, since all inhibited $P$. fluorescens and all but one inhibited St. xylosus. Overall, the L. hilgardii natural isolates gave almost twice the number of positive results compared with L. plantarum (26 vs. 14).
Freeze-dried supernatants $(5 \mathrm{mg})$ of wild strains grown in MRS medium showed antimicrobial activity which disappeared when treated with proteinase $\mathrm{K}$, thus indicating the proteinaceous nature of the inhibitory substance. Interestingly, contrary to all other strains, DSM 20174 supernatant from the MRS culture did not produce any inhibitory activity on the plate disk test, indicating that the antimicrobial activity shown by the DSM 20174 extract is determined by the fermented chicory matrix. To better investigate the strength of the inhibitory activity, we incubated the most susceptible strains, namely Listeria innocua, P. fluorescens and St. xylosus, in liquid cultures in the presence of 2 and $4 \mathrm{mg}$ of extract from chicory fermented by L. plantarum DSM 20174. The growth curves, determined over a period of $24 \mathrm{~h}$ of incubation at $30^{\circ} \mathrm{C}$, are presented in Fig. 1. In all cases, the growth dynamics of all strains (measured as the increase of turbidity of the cultures) was negatively affected by the presence of the extracts, even at the lower concentrations, although at different rates. When $4 \mathrm{mg}$ was used, the growth appeared to be highly impaired. Although the antimicrobial activity was not evident in the absence of concentration of the extract, chicory

Table 4 Antimicrobial activity of freeze-dried culture supernatants based on the plate disk test results

\begin{tabular}{|c|c|c|c|c|c|c|}
\hline Sample & Pseudomonas fluorescens & Escherichia coli & B. amyloliquefaciens & Bacillus subtilis & Staphylococcus xylosus & Listeria innocua \\
\hline Control & - & - & - & - & - & - \\
\hline \multicolumn{7}{|l|}{ L. hilgardii } \\
\hline DSM 20176 & +++ & ++ & +++ & + & ++ & ++ \\
\hline TGMMRS06 & + & - & - & - & + & - \\
\hline TGMMRS08 & + & + & + & - & + & + \\
\hline TGMMRS11 & + & - & - & + & + & - \\
\hline TGMMRS12 & + & + & + & + & + & - \\
\hline TGMMRS13 & + & - & - & - & + & - \\
\hline TGMMRS25 & + & - & - & - & + & - \\
\hline TGMMRS27 & + & - & - & - & - & - \\
\hline TGMMRS35 & + & - & - & + & + & - \\
\hline TGMMRS41 & + & - & - & + & + & - \\
\hline \multicolumn{7}{|l|}{ L. plantarum } \\
\hline DSM 20174 & +++ & ++ & +++ & ++ & ++ & ++ \\
\hline Т30РСР01 & + & + & + & + & + & + \\
\hline Т30РCP05 & - & - & - & - & - & - \\
\hline Т30РCР06 & - & + & - & - & + & + \\
\hline Т30РСР07 & - & - & - & - & + & - \\
\hline Т30РCР14 & - & - & - & - & - & - \\
\hline Т4MPCP69 & - & - & - & - & - & - \\
\hline T4MPCP71 & - & - & - & - & - & - \\
\hline T4MPCP73 & - & + & - & + & - & + \\
\hline T4MPCP77 & - & - & - & - & - & - \\
\hline T4MPCP78 & - & - & - & - & + & - \\
\hline
\end{tabular}

+++ , Inhibition halo $>20 \mathrm{~mm} ;++$, inhibition halo $<20 \mathrm{~mm} ;+$, inhibition halo $<10 \mathrm{~mm}$ 

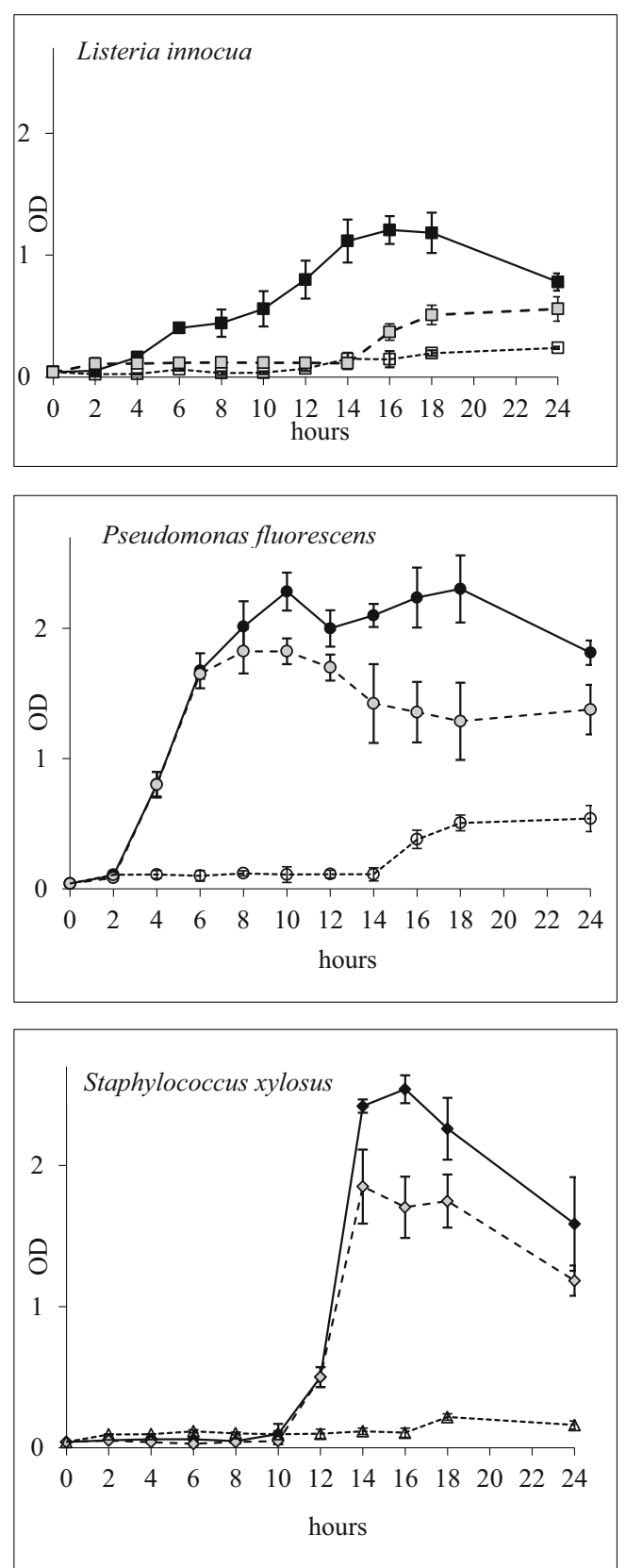

Fig. 1 Effects of the addition of chicory extracts on the growth kinetics of indicator strains. Continuous lines No chicory extract, dashed lines $2 \mathrm{mg} / \mathrm{ml}$ chicory extract, dotted lines $4 \mathrm{mg} / \mathrm{ml}$ chicory extract

fermentation would appear to be able to reduce pathogen growth.

\section{Antioxidant activity}

The DPPH scavenging activity of the samples was calculated (Tables 2, 3) using $5 \mathrm{mg}$ of freeze-dried samples. The fermented samples showed variable behaviors: in those inoculated with $L$. plantarum two strains showed activity nearly as low as that of the control, while the other samples showed quenching activities which were at least threefold higher
(22-26\%). The type strain DSM 20174 gave the highest values, close to a fivefold increase in scavenging activity, which was slightly higher than that present at the beginning of the experiment.

With respect to L. hilgardii isolates, their quenching activity was revealed to be much lower than those of the L. plantarum strains. In particular, three strains did not show any detectable activity, while the remaining showed very low levels, comparable with those of the non-inoculated control, with the sole exception of strain TGMMRS25 that reached $18.12 \%$. On the contrary, the type strain gave a high value, similar to that produced by the type strain of $L$. plantarum.

In brief, our results indicate that the total antioxidative activity of a fermented plant-based product cannot be predicted on the basis of its total phenolic content alone-rather the synergism between polyphenolic compounds and microbial strains has to be taken into account, as suggested by Naczk and Shahidi (2006).

\section{Phenolic acids profile}

Preliminary data on total phenolic content of the noninoculated pasteurized control samples after $48 \mathrm{~h}$ of fermentation showed $132 \pm 8 \mathrm{GAE} / \mathrm{mg}$ of freeze-dried sample, whereas for the L. plantarum and L. hilgardii type strain samples, the average phenolic content was $476 \pm 43$ and $492 \pm 51$ $\mathrm{GAE} / \mathrm{mg}$, respectively, evidencing a higher presence of compounds in the fermented homogenates. Our data are in agreement with those of Hur et al. (2014), thereby confirming the capability of these strains to increase the release of flavonoids during growth.

To evaluate the effect of the strain on the release of polyphenols, we determined the HPLC profile of the phenolic acids in all fermented samples and in the non-inoculated control after $48 \mathrm{~h}$. HPLC analysis of all inoculated samples produced a similar chromatographic pattern. A typical example of the chromatogram of a sample fermented with the L. plantarum DSM 20174 type strain is reported in Fig. 2.

It is interesting to note the presence of peak 1, identified as gallic acid; this peak was present in the chicory sample fermented with the L. plantarum DSM 20174 type strain but was not detected in the non-inoculated control, which contained only protocatechic acid (peak 2 ). This finding could be related to microbial tannase activity which catalyzes the hydrolysis of ester bonds into hydrolyzable tannins, releasing gallic acid, as suggested by Rodríguez et al. (2008) and Rodríguez et al. (2009) for strains of $L$. plantarum. Peaks 3 and peak 4 were identified as chicoric and chlorogenic acid, respectively, confirming that chicories contain good amounts of these compounds, as also previously reported by Rossetto et al. (2005) and Heimler et al. (2009). The numerous small peaks appearing between peaks 2 and 3 could be the product of degradation of phenolic compounds, since it is known that 


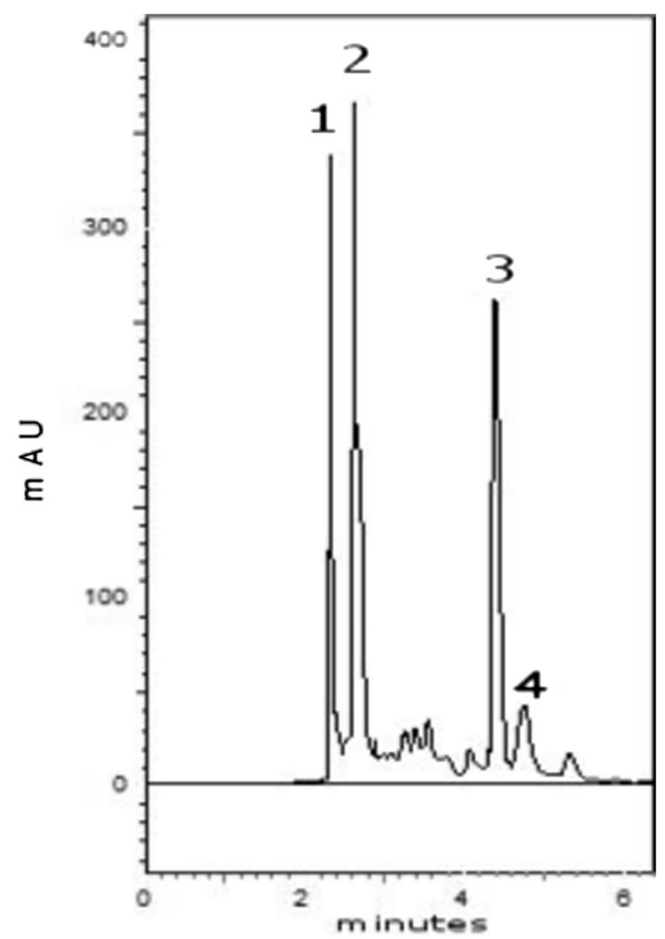

Fig. 2 Representative high-performance liquid chromatography chromatogram of chicory samples fermented with Lactobacillus plantarum DSM 20174. Peaks: 1 Gallic acid, 2 protocatechic acid, 3 chicoric acid, 4 chlorogenic acid

strains of LAB possess enzymes capable of breaking down higher molecular weight phenolic compounds (Filannino et al. 2015). Consequently,, we conducted a preliminary characterization of these smaller peaks by testing the fractions collected from the extract and searching for a correspondence between phenolic profiles and antioxidant capacity. The extracts were solubilized in distilled water, and the antiradical activity of the fractions was determined. The ability to scavenge the DPPH free radicals was present in those fractions containing peaks 1 , 2,3 and 4 , but no activity was detected in the pooled fraction containing the small peaks between peaks 2 and 3 .

It has been reported that some strains of $L$. plantarum possess tannase and decarboxylases which enable the degradation of phenolic compounds found in foods (Rodríguez et al. 2008, 2009; Jiménez et al. 2014). The presence of tannase in strains $L$. plantarum and $L$. hilgardii could be advantageous to these microbes as they are found abundantly on plant material, which is known to possess relevant amounts of polyphenols. It is feasible that these bacterial strains have learned to withstand their toxicity and to transform them into nutrient sources. The final products of tannase activity are gallic acid and glucose derived from the glycosylated compounds. To better determine the possible microbial involvement on gallic acid production, we measured glucose content in noninoculated samples at the beginning of fermentation and after $48 \mathrm{~h}$ of fermentation and in samples fermented with
L. plantarum and L. hilgardii type strains (Table 2). Samples with $L$. plantarum and L. hilgardii showed considerably higher residual glucose, which could be released from the microbial degradation of glucose-containing polyphenols. In addition, the conversion of glycosides into their aglycone forms by fermentation could be a means of increasing antioxidative activity, as suggested by Tsangalis et al. (2002).

According to Hur et al. (2014) fermentation could represent a good strategy for production or extraction of antioxidant active compounds from natural sources, and new bioactive compounds could be generated during fermentation.

\section{Conclusions}

We reported that several microbial species can grow on chicory, with $L$. plantarum and L. hilgardii giving the best performances among those tested, and also can provide the fermented substrate with antioxidant and antimicrobial activities. After $48 \mathrm{~h}$ of microbial growth, plant-fermented products showed increased antioxidant activity due to the release of phenolic compounds. Moreover, the observed antimicrobial activity was also a result of bacterial growth, since it was found only in fermented samples.

In conclusion, the identification and exploitation of suitable microbial strains for the fermentation process could be used to design functional food from plants historically considered as folk medicine. The potential health benefits of such functional food are open to investigation and would be easier to demonstrate if epidemiological data on the absence of toxicity were available. To our knowledge, our study is the first to examine microbiological exploitation of red chicory by-products with the aim to obtain a fermented product with added beneficial attributes.

Acknowledgments The authors wish to thank F. Fontana and S. Zannoni for their skillful technical assistance.

\section{Ethics statement}

Financial statement This study was funded in part by POR "Competitività regionale e occupazione" - parte FESR 2007/2013 Azione 1.1.1. Progetto "RISIB" SMUPR n. 4145 "Potenziamento della rete di infrastrutture a supporto dell'innovazione biotecnologica" and by MIUR (ex-60\% grant).

Conflict of interest The authors declare that they have no conflict of interest.

\section{References}

Alberto MR, Farias ME, Manca de Nadra MC (2001) Effect of gallic acid and catechin on Lactobacillus hilgardii $5 \mathrm{w}$ growth and metabolism of organic compounds. J Agric Food Chem 49:4359-4363 
Arvanitoyannis IS, Kassaveti A (2007) Current and potential uses of composted olive oil waste. Int J Food Sci Tech 42:281-295

Bovo B, Fontana F, Giacomini A, Corich V (2011) Effects of yeast inoculation on volatile compound production by grape marcs. Ann Microbiol 61:117-124

Drosinos EH, Paramithiotis S, Kolovos G, Tsikouras I, Metaxopoulos I (2007) Phenotypic and technological diversity of lactic acid bacteria and staphylococci isolated from traditionally fermented sausages in Southern Greece. Food Microbiol 24:260-267

Escarpa A, Gonzales MC (2001) Approach to the content of total extractable phenolic compounds from different food samples by comparison of chromathographic and spectrophotometric methods. Anal Chim Acta 427:119-127

Ferioli F, Manco MA, Antuono LF (2015) Variation of sesquiterpene lactones and phenolics in chicory and endive germplasm. J Food Comp Anal 39:77-86

Filannino P, Baia Y, Di Cagno R, Gobbetti M, Gänzle MG (2015) Metabolism of phenolic compounds by Lactobacillus spp. during fermentation of cherry juice and broccoli puree. Food Microbiol 46: 272-279

Finotti E, Gezzi R, Nobili F, Garaguso I, Friedman M (2015) Effect of apple, baobab, red-chicory, and pear extracts on cellular energy expenditure and morphology of a Caco-2 cells using transepithelial electrical resistance (TEER) and scanning electron microscopy (SEM). RSC Adv 5:22490-22498

Gardner NJ, Savard T, Obermeier P, Caldwell G, Champagne CP (2001) Selection and characterization of mixed starter cultures for lactic acid fermentation of carrot, cabbage, beet and onion vegetable mixtures. Int J Food Microbiol 64:261-275

Giacomini A, Squartini A, Nuti MP (2000) Nucleotide Sequence and Analysis of Plasmid pMD136 from Pediococcus pentosaceus FBB61 (ATCC43200) Involved in Pediocin A Production. Plasmid 43:111-122

Innocenti M, Gallori S, Giaccherini C, Ieri F, Vincieri FF, Mulinacci N (2005) Evaluation of the phenolic content in the aerial parts of different varieties of Cichorium intybus L. J Agric Food Chem 53: 6497-6502

Heimler D, Isolani L, Vignolini P, Romani A (2009) Polyphenol content and antiradical activity of Cichorium intybus L. from biodynamic and conventional farming. Food Chem 114:765-760

Hur SJ, Lee SY, Kim YC, Choi I, Kim GB (2014) Effect of fermentation on the antioxidant activity in plant-based food. Food Chem 160: 346-356

Jiménez N, Esteban-Torres M, Mancheño JM, de las Rivas B, Muñoza R (2014) Tannin degradation by a novel tannase enzyme present in some Lactobacillus plantarum strains. App Environ Microbiol 40: 2991-2997

Koukounaras A, Siomos AS (2010) Changes in antioxidant activity of radicchio during storage. Acta Hortic 877:1281-1286

Lante A, Nardi T, Zocca F, Giacomini A, Corich V (2011) Evaluation of red chicory extract as a natural antioxidant by pure lipid oxidation and yeast oxidative stress response as model systems. J. Agric Food Chem 59:5318-5324

Leroy F, De Vuyst L (2004) Lactic acid bacteria as functional starter cultures for the food fermentation industry. Trends Food Sci Technol 15:67-78
Maragkoudakis PA, Nardi T, Bovo B, D'Andrea M, Howell KS, Giacomini A, Corich V (2013) Biodiversity, dynamics and ecology of bacterial community during grape marc storage for the production of grappa. Int J Food Microbiol 162:143-151

Martìnez-Muñoz GA, Kane P (2008) Vacuolar and plasma membrane proton pumps collaborate to achieve cytosolic $\mathrm{pH}$ homeostasis in yeast. J Biol Chem 283:20309-20319

Miliauskas G, Venskutonis P, van Beek T (2004) Screening of radical scavenging activity of some medicinal and aromatic plant extracts. Food Chem 85:231-237

Miller BJ, Franz CM, Cho GS, Du Toit M (2011) Expression of the malolactic enzyme gene (mle) from Lactobacillus plantarum under winemaking conditions. Curr Microbiol 62:1682-1688

Moreno-Arribas M, Polo MC, Jorganes F, Muñoz R (2003) Screening of biogenic amine production by lactic acid bacteria isolated from grape must and wine. Int J Food Microbiol 84:117-123

Naczk M, Shahidi F (2006) Phenolics in cereals, fruits and vegetables: occurrence, extraction and analysis. J Pharmaceut Biomed 41:15231542

Ribéreau-Gayon P, Dubourdieu D, Donèche B, Lonvaud A (2006) Handbook of Enology: The microbiology of wine and vinifications, vol. 1, 2nd edn. Wiley, New York

Rodas A, Ferrer S, Pardo I (2005) Polyphasic study of wine Lactobacillus strains: taxonomic implications. Int J Syst Evol Micr 55:197-207

Rodríguez H, de Las RB, Gómez-Cordovés C, Muñoz R (2008) Characterization of tannase activity in cell-free extracts of Lactobacillus plantarum CECT $748^{\mathrm{T}}$. Int J Food Microbiol 121: 92-98

Rodríguez H, Curiel JA, Landete JM, de Las RB, de Felipe FL, GómezCordovés C, Muñoz R (2009) Food phenolics and lactic acid bacteria. Int J Food Microbiol 132:79-90

Rossetto M, Lante A, Vanzani P, Spettoli P, Scarpa M, Rigo A (2005) Red chicories as potent scavengers of highly reactive radicals: A study on their phenolic composition and peroxyl radical trapping capacity and efficiency. J Agric Food Chem 53:8169-8175

Ruiz-Barba JL, Cathcart DP, Warner PJ, Jiménez-Díaz R (1994) Use of Lactobacillus plantarum LPCO10, a bacteriocin producer, as a starter culture in Spanish-style green olive fermentations. Appl Environ Microbiol 60:2059-2064

Singleton VL, Orthofer R, Lamuela-Raventos RM (1999) Analysis of total phenols and other oxidation substrates and antioxidants by means of Folin-Ciocalteu reagent. Method Enzymol 299:152-178

Spyropoulos B, Misiakos E, Fotiadis C, Stoidis C (2011) Antioxidant properties of probiotics and their protective effects in the pathogenesis of radiation induced enteritis and colitis. Digest Dis Sci 56:285294

Street RA, Sidana J, Prinsloo G (2013) Cichorium intybus: traditional uses, phytochemistry, pharmacology, and toxicology. Evid Based Complement Alternat Med 2013:579319

Tsangalis D, Ashton JF, McGill AEJ, Shah NP (2002) Enzymic transformation of isoflavone phytoestrogens in soymilk by b-glucosidase producing bifidobacteria. J Food Sci 67:3104-3113

Vuyst LD, Vandamme EJ (1994) Bacteriocins of lactic acid bacteria: microbiology, genetics and applications. Blackie Academic \& Professional, London

Zuleta A, Sambucetti ME (2001) Inulin determination for food labelling. J Agric Food Chem 49:4750-4572 